\title{
Int-Soft Filters in Hoops
}

\author{
R. A. Borzooei ${ }^{1}$, M. Sabetkish ${ }^{1}$, E. H. Roh ${ }^{2}$, and M. Aaly Kologani ${ }^{3}$ \\ ${ }^{1}$ Department of Mathematics, Shahid Beheshti University, Tehran, Iran \\ ${ }^{2}$ Department of Mathematics Education, Chinju National University of Education, Jinju, Korea \\ ${ }^{3}$ Hatef Higher Education Institute, Zahedan, Iran
}

\section{ljfis}

\begin{abstract}
In this paper, we introduce the notion of int-soft filters in hoops and related properties are investigated. Characterizations of int-soft filters are discussed and we introduce a congruence relation by using int-soft filter and we show that the quotient is a hoop. Also, we introduce the notions of int-soft $\mathrm{n}$-fold (positive) implicative and int-soft $\mathrm{n}$-fold fantastic filters of hoops, we study some equivalence definitions of them and the relation between them, we prove that every int-soft $n$-fold positive implicative filter is an int-soft fantastic filter and int-soft implicative filter. Also, we investigate about the quotient structure that is made by them.
\end{abstract}

Keywords: Hoop, $n$-fold implicative (positive implicative, fantastic) filter, Soft set, Int-soft filter, $\gamma$-inclusive set.

\section{Introduction}

Non-classical logic has become a considerable formal tool for computer science and artificial intelligence to deal with fuzzy information and uncertainty information. Many-valued logic, a great extension and development of classical logic, has always been a crucial direction in nonclassical logic. In order to research the many-valued logical system whose propositional value is given in a lattice, Bosbach [1, 2] proposed the concept of hoops, and discussed their some properties. Hoops are naturally ordered commutative residuated integral monoids. In the last years, hoops theory was enriched with deep structure theorems [1-3]. Many of these results have a strong impact with fuzzy logic. Particularly, from the structure theorem of finite basic hoops [3, Corollary 2.10], one obtains an elegant short proof of the completeness theorem for propositional basic logic [3, Theorem 3.8], introduced by Hajek [4]. The algebraic structures

Received: May 20, 2019

Revised : Sep. 17, 2019

Accepted: Sep. 20, 2019

Correspondence to: E. H. Roh

(ehroh9988@gmail.com)

(CThe Korean Institute of Intelligent Systems

cc) This is an Open Access article distributed under the terms of the Creative Commons Attribution Non-Commercial License (http://creativecommons.org/licenses/ by-nc/3.0/] which permits unrestricted noncommercial use, distribution, and reproduction in any medium, provided the original work is properly cited. corresponding to Hajek's propositional (fuzzy) basic logic, BL-algebras, are particular cases of hoops. The main example of BL-algebras in interval $[0,1]$ endowed with the structure induced by a t-norm. MV-algebras, product algebras and Gödel algebras are the most known classes of BL-algebras. Recent investigations are concerned with non-commutative generalizations for these structures. During these years, many researchers study on hoops in different way, and got some results on hoops [5]. Borzooei and Kologani [6] investigated different kind of filters on hoops such as (positive)implicative and fantastic filters, and studied the relation between them and investigated congruence relation that is made by them and studied about the quotients structure.

Molodtsov [7] introduced the concept of soft set as a new mathematical tool for dealing with uncertainties that is free from the difficulties that have troubled the usual theoretical approaches. Molodtsov pointed out several directions for the applications of soft sets. At 
present, works on the soft set theory are progressing rapidly. Maji et al. [8] described the application of soft set theory to a decision making problem. Maji et al. [9] also studied several operations on the theory of soft sets. Chen et al. [10] presented a new definition of soft set parametrization reduction, and compared this definition to the related concept of attributes reduction in rough set theory. The algebraic structure of set theories dealing with uncertainties has been studied by some authors. Feng $[8]$ considered the application of soft rough approximations in multicriteria group decision-making problems. Aktas and Cagman [12] studied the basic concepts of soft set theory, and compared soft sets to fuzzy and rough sets, providing examples to clarify their differences. They also discussed the notion of soft groups. After than, many algebraic properties of soft sets are studied [13-16]. In this paper, we introduce the notion of int-soft filters in hoops and related properties are investigated. Characterizations of int-soft filters are discussed and we introduce a congruence relation by using int-soft filter and we show that the quotient is a hoop. Also, we introduce the notions of int-soft n-fold (positive) implicative and int-soft $\mathrm{n}$-fold fantastic filters of hoops, we study some equivalence definitions of them and the relation between them, we prove that every int-soft $\mathrm{n}$-fold positive implicative filter is an int-soft fantastic filter and int-soft implicative filter. Also, we investigate about the quotient structure that is made by them.

\section{Preliminaries}

In this section, recollect some definitions an results which will be used in this paper

Definition 2.1 ( [1, 2]). A hoop is an algebra $(H, \odot, \rightarrow, 1)$ of type $(2,2,0)$ such that, for all $x, y, z \in H$,

(HP1) $(H, \odot, \rightarrow, 1)$ is a commutative monoid.

(HP2) $x \rightarrow x=1$.

(HP3) $(x \odot y) \rightarrow z=x \rightarrow(y \rightarrow z)$.

(HP4) $x \odot(x \rightarrow y)=y \odot(y \rightarrow x)$.

On hoop $H$, we define $x \leq y$ if and only if $x \rightarrow y=1$. It is easy to see that $\leq$ is a partial order relation on $H$. A hoop $H$ is called bounded if there is an element $0 \in H$ such that, for all $x \in H, 0 \leq x$. Let $H$ be a bounded hoop. For all $x \in H$, we define a negation ' on $H$ by $x^{\prime}=x \rightarrow 0$. If, for all $x \in H$, $x^{\prime \prime}=x$, then the bounded hoop $H$ is said to have the double negation property, (DNP), for short.

The following proposition provides some properties of hoop.
Proposition 2.2 ( [1, 2]). Let $H$ be a hoop. Then, for all $x, y, z \in H$, the following properties hold,

(i) $x \odot y \leq z$ if and only if $x \leq y \rightarrow z$.

(ii) $x \leq y$ if and only if $x \rightarrow y=1$.

(iii) $x \odot y \leq x, y$.

(iv) $x \leq y \rightarrow x$.

(v) $x \leq(x \rightarrow y) \rightarrow y$.

(vi) $x \rightarrow 1=1$ and $1 \rightarrow x=x$.

(vii) If $x \leq y$, then $z \rightarrow x \leq z \rightarrow y, y \rightarrow z \leq x \rightarrow z$ and $x \odot z \leq y \odot z$.

(viii) $x \rightarrow y \leq(y \rightarrow z) \rightarrow(x \rightarrow z)$.

Definition 2.3 ( [3, 17]). Let $H$ be a hoop and $\emptyset \neq F \subseteq H$ such that $1 \in F$. Then, for any $x, y, z \in H$, and $n \in \mathbb{N}, F$ is called (i) a filter of $H$, if $x, x \rightarrow y \in F$, then $y \in F$.

(ii) an $n$-fold implicative filter of $H$, if $x^{n} \rightarrow(y \rightarrow z) \in F$ and $x^{n} \rightarrow y \in F$, then $x^{n} \rightarrow z \in F$.

(iii) an $n$-fold positive implicative filter of $H$, if $x \rightarrow\left(\left(y^{n} \rightarrow\right.\right.$ $z) \rightarrow y) \in F$ and $x \in F$, then $y \in F$.

(iv) an $n$-fold fantastic filter of $H$, if $z \in F$ and $z \rightarrow(y \rightarrow$ $x) \in F$, then $\left(\left(x^{n} \rightarrow y\right) \rightarrow y\right) \rightarrow x \in F$.

Definition 2.4 ( [7]). Let $U$ be an initial universe set, $E$ be a set of parameters, $\mathcal{P}(U)$ be the power set of $U$ and $\emptyset \neq A \subseteq E$. Then a soft set $(\alpha, A)$ over $U$ is defined to be the set of ordered pairs $(\alpha, A)=\{(x, \alpha(x)) \mid x \in A, \alpha(x) \in \mathcal{P}(U)\}$, where $\alpha: A \rightarrow \mathcal{P}(U)$. For a soft set $(\alpha, A)$ over $U$ and a subset $\gamma$ of $U$, the $\gamma$-inclusive set of $(\alpha, A)$, denoted by $i_{H}(\alpha, \gamma)$, is defined to be the set

$$
i_{H}(\alpha, \gamma):=\{x \in A \mid \gamma \subseteq \alpha(x)\} .
$$

Notation. From now on, in this paper $(H, \odot, \rightarrow, 1)$ or simply $H$ is a hoop, unless otherwise state.

\section{Int-Soft Filters}

In this section, we introduce the notion of int-soft filter of hoops and find some equivalence definitions of that. Also, we define a congruence relation by int-soft filter of $H$ and show that the quotient, that is made by it, is a hoop.

Definition 3.1. A soft set $(\alpha, H)$ is called an int-soft filter of $H$ over $U$ if, for all $\gamma \in \mathcal{P}(U), \gamma$-inclusive $\operatorname{set} \emptyset \neq i_{H}(\alpha, \gamma)$ of $(\alpha, H)$ is a filter of $H$.

Example 3.2. Let $H=\{0, a, b, c, 1\}$. Then, for any $x, y \in H$, 
define the operations $\odot$ and $\rightarrow$ on $H$ as follows:

\begin{tabular}{|c|c|c|c|c|c|c|c|c|c|c|}
\hline$\rightarrow$ & 0 & $\mathrm{a}$ & b & $\mathrm{c}$ & 1 & $\odot$ & 0 & $\mathrm{a}$ & b & $\mathrm{c}$ \\
\hline 0 & 1 & 1 & 1 & 1 & 1 & 0 & 0 & 0 & 0 & 0 \\
\hline $\mathrm{a}$ & b & 1 & b & 1 & 1 & $\mathrm{a}$ & 0 & $\mathrm{a}$ & 0 & $\mathrm{a}$ \\
\hline b & $\mathrm{a}$ & $\mathrm{a}$ & 1 & 1 & 1 & b & 0 & 0 & b & $b$ \\
\hline $\mathrm{c}$ & 0 & $\mathrm{a}$ & b & 1 & 1 & $c$ & 0 & $\mathrm{a}$ & b & c \\
\hline 1 & 0 & $\mathrm{a}$ & $\mathrm{b}$ & $\mathrm{c}$ & 1 & 1 & 0 & $\mathrm{a}$ & $\mathrm{b}$ & $\mathrm{c}$ \\
\hline
\end{tabular}

Then by routine calculations, we can see that $(H, \odot, \rightarrow, 0,1)$ is a bounded hoop. Let $(\lambda, H)$ be a soft set over $U=\mathbb{Z}$ in $H$ such that $\alpha: H \rightarrow P(U)$ and $\alpha(1)=\mathbb{Z}, \alpha(a)=2 \mathbb{Z}, \alpha(b)=$ $4 \mathbb{Z}, \alpha(0)=4 \mathbb{Z}$ and $\alpha(c)=\mathbb{Z}$. Then $(\alpha, H)$ is an int-soft filter of $H$ over $U$.

Theorem 3.3. Let $(\alpha, H)$ be a soft set of $H$. Then, for any $x, y, z \in H$, the following statements are equivalent:

(i) $(\alpha, H)$ is an int-soft filter of $H$ over $U$,

(ii) $\alpha(x) \subseteq \alpha(1)$ and $\alpha(x) \cap \alpha(x \rightarrow y) \subseteq \alpha(y)$,

(iii) $\alpha(x) \subseteq \alpha(1)$ and $\alpha(x \rightarrow y) \cap \alpha(y \rightarrow z) \subseteq \alpha(x \rightarrow z)$,

(iv) $\alpha(x) \subseteq \alpha(1)$ and $\alpha(x \rightarrow y) \cap \alpha(x \odot z) \subseteq \alpha(y \odot z)$,

(v) if $x \leq y \rightarrow z$, then $\alpha(x) \cap \alpha(y) \subseteq \alpha(z)$.

Proof. (i) $\Rightarrow$ (ii) Suppose $(\alpha, H)$ is an int-soft filter of $H$ over $U$. Then, for any $x, y \in H, \alpha(x \rightarrow y) \cap \alpha(x)=\gamma$. Thus, $\gamma \subseteq \alpha(x)$ and $\gamma \subseteq \alpha(x \rightarrow y)$, and so $x \in i_{H}(\alpha, \gamma)$ and $x \rightarrow y \in i_{H}(\alpha, \gamma)$. Since $i_{H}(\alpha, \gamma)$ is a filter of $H$ and $x \odot(x \rightarrow$ $y) \in i_{H}(\alpha, \gamma)$, by Proposition 2.2(v), $x \odot(x \rightarrow y) \leq y$, and so $y \in i_{H}(\alpha, \gamma)$. Hence, $\gamma=\alpha(x) \cap \alpha(x \rightarrow y) \subseteq \alpha(y)$. Now, let $x \in H$ and $\alpha(x)=\gamma_{x}$. Then $x \in i_{H}(\alpha, \gamma)$. Since $i_{H}(\alpha, \gamma)$ is a filter of $H, 1 \in i_{H}(\alpha, \gamma)$ and $\gamma_{x}=\alpha(x) \subseteq \alpha(1)$. Hence, $\alpha(x) \subseteq \alpha(1)$.

(ii) $\Rightarrow$ (iii) It is clear that, for any $x \in H, \alpha(x) \subseteq \alpha(1)$. By Proposition 2.2 (viii), for any $x, y, z \in H, x \rightarrow y \leq(y \rightarrow$ $z) \rightarrow(x \rightarrow z)$, and so

$$
(x \rightarrow y) \rightarrow[(y \rightarrow z) \rightarrow(x \rightarrow z)]=1 .
$$

By (ii), we have

$$
\begin{aligned}
& \alpha(x \rightarrow y) \cap \alpha(y \rightarrow z) \\
& =\alpha(x \rightarrow y) \cap \alpha(1) \cap \alpha(y \rightarrow z) \\
& =\alpha(x \rightarrow y) \cap \alpha((x \rightarrow y) \\
& \quad \rightarrow[(y \rightarrow z) \rightarrow(x \rightarrow z)]) \cap \alpha(y \rightarrow z) \\
& \subseteq \alpha((y \rightarrow z) \rightarrow(x \rightarrow z)]) \cap \alpha(y \rightarrow z) \\
& \subseteq \alpha(x \rightarrow z) .
\end{aligned}
$$

Hence, $\alpha(x \rightarrow y) \cap \alpha(y \rightarrow z) \subseteq \alpha(x \rightarrow z)$.

(iii) $\Rightarrow$ (iv) Let $x, y, z \in H$. Since $y \odot z \leq y \odot z$, we have $y \leq z \rightarrow(y \odot z)$. Then by Proposition 2.2(vii), $x \rightarrow y \leq x \rightarrow$ $(z \rightarrow(y \odot z))$, and so $x \rightarrow y \leq(x \odot z) \rightarrow(y \odot z)$. Thus, $(x \rightarrow y) \rightarrow((x \odot z) \rightarrow(y \odot z))=1$. Hence, by (iii), we get that

$$
\begin{aligned}
& \alpha(1 \rightarrow(x \rightarrow y)) \cap \alpha((x \rightarrow y) \\
& \quad \rightarrow((x \odot z) \rightarrow(y \odot z))) \cap \alpha(x \odot z) \\
& \subseteq \alpha((x \odot z) \rightarrow(y \odot z)) \cap \alpha(x \odot z) \\
& =\alpha(1 \rightarrow(x \odot z)) \cap \alpha((x \odot z) \rightarrow(y \odot z)) \\
& \subseteq \alpha(1 \rightarrow(y \odot z)) \\
& =\alpha(y \odot z) .
\end{aligned}
$$

Hence, $\alpha(x \rightarrow y) \cap \alpha(y \odot z) \subseteq \alpha(x \odot z)$.

(iv) $\Rightarrow$ (v) Let $x, y, z \in H$ such that $x \leq y \rightarrow z$. It is clear that $x \rightarrow(y \rightarrow z)=1$. Then by (iv), we have,

$$
\begin{aligned}
& \alpha(x) \cap \alpha(y) \cap \alpha(x \rightarrow(y \rightarrow z)) \subseteq \alpha(y) \cap \alpha(y \rightarrow z) \\
& =\alpha(1 \odot y) \cap \alpha(y \rightarrow z) \subseteq \alpha(1 \odot z)=\alpha(z) .
\end{aligned}
$$

(v) $\Rightarrow$ (i) Let $x \in i_{H}(\alpha, \gamma)$. Then $\gamma \subseteq \alpha(x)$. Since, for any $x \in H, x \rightarrow(x \rightarrow 1)=1$, we have $\alpha(x)=\alpha(x) \cap$ $\alpha(x) \subseteq \alpha(1)$. Hence, for any $x \in i_{H}(\alpha, \gamma), \alpha(x) \subseteq \alpha(1)$. Now, suppose for any $x, y \in H, x, x \rightarrow y \in i_{H}(\alpha, \gamma)$. Then $\gamma \subseteq \alpha(x) \cap \alpha(x \rightarrow y)$. Since $(x \odot(x \rightarrow y)) \rightarrow y=x \rightarrow$ $((x \rightarrow y) \rightarrow y)=1$ by (v), we have $\alpha(x) \cap \alpha(x \rightarrow y) \subseteq \alpha(y)$ and so $\gamma \subseteq \alpha(y)$. Hence, $y \in i_{H}(\alpha, \gamma)$. Therefore, $(\alpha, H)$ is an int-soft filter of $H$.

Corollary 3.4. Let $H$ be a bounded hoop and $(\alpha, H)$ be an int-soft filter of $H$. Then, for any $x, y, z \in H$, the following sentences hold:

(i) If $x \leq y$, then $\alpha(x) \subseteq \alpha(y)$.

(ii) $\alpha(x \odot y) \subseteq \alpha(x) \cap \alpha(y)$.

(iii) $\alpha\left(x^{\prime}\right) \subseteq \alpha(x \rightarrow y)$.

(iv) $\alpha((x \rightarrow y) \rightarrow y) \cap \alpha(x) \subseteq \alpha(y)$.

Proof. (i) Let $x, y \in H$ such that $x \rightarrow y=1$. Since $(\alpha, H)$ is an int-soft filter of $H$, we have $\alpha(x) \cap \alpha(x \rightarrow y) \subseteq \alpha(y)$, and so $\alpha(x) \subseteq \alpha(y)$. By (i) and Proposition 2.2. the proof of other cases is clear.

Proposition 3.5. Let $(\alpha, H)$ be a soft set of $H$ and for any $x \in H, x^{2}=x$. Then $(\alpha, H)$ is an int-soft filter of $H$ over $U$ 
if and only if $\alpha(x) \subseteq \alpha(1)$ and

$$
\alpha((x \odot y) \rightarrow z) \cap \alpha(x \rightarrow y) \subseteq \alpha(x \rightarrow z) .
$$

Proof. Let $x, y \in H$. Since $((1 \odot x) \rightarrow y)=x \rightarrow y$ by assumption, we have $\alpha((1 \odot x) \rightarrow y) \cap \alpha(1 \rightarrow x) \subseteq \alpha(1 \rightarrow y)$ and so $\alpha(x) \cap \alpha(x \rightarrow y) \subseteq \alpha(y)$. Hence, $(\alpha, H)$ is an int-soft filter of $H$ over $U$.

Conversely, let $x, y, z \in H$. Then by Proposition 2.1.(viii),

$$
(x \rightarrow y) \odot(y \rightarrow(x \rightarrow z)) \leq x \rightarrow(x \rightarrow z)
$$

Since, for any $x \in H, x^{2}=x$, we have $(x \rightarrow y) \odot(y \rightarrow$ $(x \rightarrow z)) \leq x \rightarrow z$. Moreover, $(\alpha, H)$ is an int-soft filter of $H$, then $\alpha(x) \subseteq \alpha(1)$ and by Corollary 3.4(ii), we have $\alpha((x \odot y) \rightarrow z) \cap \alpha(x \rightarrow y) \subseteq \alpha(x \rightarrow z)$

Theorem 3.6. Let $x, y \in H$ and $(\alpha, H)$ be an int-soft filter of $H$. Then we define a binary relation $\equiv$ on $H$ by $x \equiv_{(\alpha, H)} y$ if and only if $\alpha(x \rightarrow y) \cap \alpha(y \rightarrow x)=\alpha(1)$. Then $\equiv_{(\alpha, H)}$ is a congruence relation on $H$.

Proof. By routine calculation it is clear that $\equiv_{(\alpha, H)}$ is reflective and symmetric. Now, we prove that $\equiv_{(\alpha, H)}$ is transitive. Let $x, y, z \in H$ such that $x \equiv_{(\alpha, H)} y$ and $y \equiv_{(\alpha, H)} z$. Then $\alpha(x \rightarrow y) \cap \alpha(y \rightarrow x)=\alpha(1)$ and $\alpha(y \rightarrow z) \cap \alpha(z \rightarrow y)=$ $\alpha(1)$ By Proposition 2.2(viii), $(x \rightarrow y) \odot(y \rightarrow z) \leq(x \rightarrow y)$. Since $(\alpha, H)$ is an int-soft filter of $H$, by Corollary 3.4(ii), we have $\alpha(x \rightarrow y) \cap \alpha(y \rightarrow z) \subseteq \alpha(x \rightarrow z)$ By the similar way, $\alpha(z \rightarrow y) \cap \alpha(y \rightarrow x) \subseteq \alpha(z \rightarrow x)$ Thus,

$$
\begin{aligned}
\alpha(1) & \subseteq \alpha(x \rightarrow y) \cap \alpha(y \rightarrow x) \cap \alpha(y \rightarrow z) \cap \alpha(z \rightarrow y) \\
& \subseteq \alpha(z \rightarrow x) \cap \alpha(x \rightarrow z),
\end{aligned}
$$

and so $\alpha(x \rightarrow z) \cap \alpha(z \rightarrow x)=\alpha(1)$. Hence, $x \equiv_{(\alpha, H)} z$. Therefore, $\equiv_{(\alpha, H)}$ is an equivalence relation. Suppose $x \equiv_{(\alpha, H)}$ $y$. We prove that for any $z \in H, x \rightarrow z \equiv_{(\alpha, H)} y \rightarrow z$. Since $x \equiv_{(\alpha, H)} y$, we have $\alpha(x \rightarrow y) \cap \alpha(y \rightarrow x)=\alpha(1)$. By Proposition 2.2(viii), $x \rightarrow y \leq(y \rightarrow z) \rightarrow(x \rightarrow z)$ and $(\alpha, H)$ is an int-soft filter of $H$, then by Corollary 3.4 (i), $\alpha(x \rightarrow$ $y) \subseteq \alpha((y \rightarrow z) \rightarrow(x \rightarrow z))$ By the similar way, $\alpha(y \rightarrow$ $x) \subseteq \alpha((x \rightarrow z) \rightarrow(y \rightarrow z))$. Then

$$
\begin{aligned}
& \alpha(1) \\
& =\alpha(x \rightarrow y) \cap \alpha(y \rightarrow x) \\
& \subseteq v \alpha((y \rightarrow z) \rightarrow(x \rightarrow z)) \cap \alpha((x \rightarrow z) \rightarrow(y \rightarrow z)) .
\end{aligned}
$$

Hence, $x \rightarrow z \equiv_{(\alpha, H)} y \rightarrow z$. By repeating this method, it is easy to see that if $x \equiv_{(\alpha, H)} y$, then $z \rightarrow x \equiv_{(\alpha, H)} z \rightarrow y$ Now, let $x \equiv_{(\alpha, H)} y$. By Proposition 2.2(vii), $x \rightarrow y \leq$ $(x \odot z) \rightarrow(y \odot z)$. Since $(\alpha, H)$ is an int-soft filter of $H$, by Corollary 3.4(i), $\alpha(x \rightarrow y) \subseteq \alpha((x \odot z) \rightarrow(y \odot z))$ and $\alpha(y \rightarrow x) \subseteq \alpha((y \odot z) \rightarrow(x \odot z))$. Then it is clear that $\alpha(1)=\alpha((x \odot z) \rightarrow(y \odot z)) \cap \alpha((y \odot z) \rightarrow(x \odot z))$. Hence, $x \odot z \equiv_{(\alpha, H)} y \odot z$. Therefore, $\equiv_{(\alpha, H)}$ is a congrunce relation on $H$.

Notation. Let define $[x]=\left\{y \in H \mid x \equiv_{(\alpha, H)} y\right\}$ and $\frac{H}{\equiv_{(\alpha, H)}}=\{[x] \mid x \in H\}$.

Theorem 3.7. Let $H$ be a hoop and $(\alpha, H)$ be an int-soft filter on $H$. Then $\left(\frac{H}{\equiv_{(\alpha, H)}}, \otimes, \rightsquigarrow,[1]\right)$ is a hoop, where, for any $[x],[y] \in \frac{H}{\equiv_{(\alpha, H)}}$, we define: $[x] \otimes[y]=[x \odot y]$ and $[x] \rightsquigarrow[y]=[x \rightarrow y]$ and $[1]=\{x \in H \mid \alpha(x)=\alpha(1)\}$.

Also, we can define an order on $\frac{H}{\equiv_{(\alpha, H)}}$ by $[x] \leq[y]$ if and only if $\alpha(x \rightarrow y)=\alpha(1)$. Then $\left(\frac{H}{\equiv_{(\alpha, H)}}, \leq\right)$ is a poset.

\section{Int-Soft n-Fold Implicative Filters}

In this section, we introduce the notion of int-soft n-fold implicative filter of hoop and investigate some properties and equivalence definitions of it. Also, we study the quotient that is made by int-soft $n$-fold implicative filters of hoop and we show that every filter of the quotient structure is an implicative filter.

Definition 4.1. A soft set $(\alpha, H)$ is an int-soft $n$-fold implicative filter of $H$ over $U$ if, for all $\gamma \in \mathcal{P}(U)$, an $\gamma$-inclusive set $\emptyset \neq i_{H}(\alpha, \gamma)$ of $(\alpha, H)$ is an $\mathrm{n}$-fold implicative filter of $H$.

The set of all int-soft n-fold implicative filters of $H$ over $U$ is denoted by $\operatorname{INI}(\mathcal{H})$.

Example 4.2. According to Example 3.2 $(\alpha, H)$ is an int soft n-fold implicative filter of $H$ over $U$.

Theorem 4.3. Every $(\alpha, H) \in \operatorname{INI}(\mathcal{H})$ is an int-soft filter of $H$.

Proof. Let $(\alpha, H) \in \operatorname{INI}(\mathcal{H})$. Then $i_{H}(\alpha, \gamma) \neq \emptyset$. Thus, there exists $x \in i_{H}(\alpha, \gamma)$. Since $i_{H}(\alpha, \gamma)$ is an $\mathrm{n}$-fold implicative filter of $H, 1 \in i_{H}(\alpha, \gamma)$. Then it is clear that $\alpha(x) \subseteq \alpha(1)$. Now, let $x, y \in H$ and $n \in \mathbb{N}$ such that $x, x \rightarrow y \in i_{H}(\alpha, \gamma)$. Suppose $\gamma=\alpha(x) \cap \alpha(x \rightarrow y)$. Since $i_{H}(\alpha, \gamma)$ is an $\mathrm{n}$-fold implicative filter and $1^{n} \rightarrow(x \rightarrow y), 1^{n} \rightarrow x \in i_{H}(\alpha, \gamma)$, we have $1^{n} \rightarrow y \in i_{H}(\alpha, \gamma)$. So, $y \in i_{H}(\alpha, \gamma)$. Thus, $i_{H}(\alpha, \gamma)$ is a filter of $H$. Hence, $(\alpha, H)$ is an int-soft filter of $H$. 
In the following example we show that the converse of Theorem 4.3 may not be true, in general.

Example 4.4. Let $H=\{0, a, b, 1\}$. Then, for any $x, y \in H$, define the operations $\odot$ and $\rightarrow$ on $H$ as follows:

\begin{tabular}{l|cccc}
$\rightarrow$ & 0 & $\mathrm{a}$ & $\mathrm{b}$ & 1 \\
\hline 0 & 1 & 1 & 1 & 1 \\
$\mathrm{a}$ & $\mathrm{a}$ & 1 & 1 & 1 \\
$\mathrm{~b}$ & 0 & $\mathrm{a}$ & 1 & 1 \\
1 & 0 & $\mathrm{a}$ & $\mathrm{b}$ & 1
\end{tabular}

\begin{tabular}{c|cccc}
$\odot$ & 0 & $\mathrm{a}$ & $\mathrm{b}$ & 1 \\
\hline 0 & 0 & 0 & 0 & 0 \\
$\mathrm{a}$ & 0 & $\mathrm{a}$ & $\mathrm{a}$ & $\mathrm{a}$ \\
$\mathrm{b}$ & 0 & $\mathrm{a}$ & $\mathrm{b}$ & $\mathrm{b}$ \\
1 & 0 & $\mathrm{a}$ & $\mathrm{b}$ & 1
\end{tabular}

Then $(H, \odot, \rightarrow, 0,1)$ is a bounded hoop. Let $(\alpha, H)$ be a soft set over $U=\mathbb{Z}$ in $H$ such that $\alpha: H \rightarrow P(U)$ and $\alpha(1)=\mathbb{Z}, \alpha(a)=4 \mathbb{Z}, \alpha(b)=2 \mathbb{Z}$ and $\alpha(0)=4 \mathbb{Z}$. Then $(\alpha, H)$ is an int-soft filter of $H$ over $U$ but it is not an int-soft n-fold implicative filter of $H$. Because $\mathbb{Z}=\alpha(1)=\alpha\left(a^{n} \rightarrow\right.$ $(a \rightarrow 0)) \cap \alpha\left(a^{n} \rightarrow a\right) \nsubseteq \alpha\left(a^{n} \rightarrow 0\right)=4 \mathbb{Z}$.

Theorem 4.5. Let $(\alpha, H)$ be an int-soft filter of $H$. Then, for any $x, y, z \in H$ and $n \in \mathbb{N}$, the following statements are equivalent:

(i) $(\alpha, H) \in I N I(\mathcal{H})$,

(ii) $\alpha\left(x^{n} \rightarrow(y \rightarrow z)\right) \cap \alpha\left(x^{n} \rightarrow y\right) \subseteq \alpha\left(x^{n} \rightarrow z\right)$,

(iii) $\alpha\left(x^{n} \rightarrow x^{2 n}\right)=\alpha(1)$,

(iv) $\alpha\left(x^{2 n} \rightarrow y\right) \subseteq \alpha\left(x^{n} \rightarrow y\right)$.

Proof. (i) $\Rightarrow$ (ii) Since $(\alpha, H) \in I N I(\mathcal{H})$, by Theorem 4.3 it is clear that $\alpha(x) \subseteq \alpha(1)$. Now, suppose $x, y, z \in H$ and $n \in \mathbb{N}$. Let $\gamma=\alpha\left(x^{n} \rightarrow(y \rightarrow z)\right) \cap \alpha\left(x^{n} \rightarrow y\right)$. Then $x^{n} \rightarrow$ $(y \rightarrow z), x^{n} \rightarrow y \in i_{H}(\alpha, \gamma)$. Since $(\alpha, H) \in I N I(\mathcal{H})$, $i_{H}(\alpha, \gamma)$ is an $\mathrm{n}$-fold implicative filter of $H$, then $x^{n} \rightarrow z \in$ $i_{H}(\alpha, \gamma)$. Thus $\gamma \subseteq \alpha\left(x^{n} \rightarrow z\right)$. Hence,

$$
\alpha\left(x^{n} \rightarrow(y \rightarrow z)\right) \cap \alpha\left(x^{n} \rightarrow y\right) \subseteq \alpha\left(x^{n} \rightarrow z\right) .
$$

(ii) $\Rightarrow$ (iii) Let $x \in H$ and $n \in \mathbb{N}$. Since $x^{2 n} \rightarrow x^{2 n}=1$, we have $\alpha(1)=\alpha\left(x^{2 n} \rightarrow x^{2 n}\right)=\alpha\left(x^{n} \rightarrow\left(x^{n} \rightarrow x^{2 n}\right)\right)$. Then by (ii),

$$
\begin{aligned}
\alpha(1) & =\alpha\left(x^{n} \rightarrow\left(x^{n} \rightarrow x^{2 n}\right)\right) \cap \alpha\left(x^{n} \rightarrow x^{n}\right) \\
& \subseteq \alpha\left(x^{n} \rightarrow x^{2 n}\right) .
\end{aligned}
$$

Also, for any $x \in H, \alpha(x) \subseteq \alpha(1)$. Then $\alpha\left(x^{n} \rightarrow x^{2 n}\right) \subseteq$ $\alpha(1)$. Hence, $\alpha\left(x^{n} \rightarrow x^{2 n}\right)=\alpha(1)$.

(iii) $\Rightarrow$ (iv) Let $x, y \in H$ and $n \in \mathbb{N}$. By Proposition2.2(viii), we have $\left(x^{n} \rightarrow x^{2 n}\right) \odot\left(x^{2 n} \rightarrow y\right) \leq x^{n} \rightarrow y$. Since $(\alpha, H)$ be an int-soft filter of $H$, by Corollary 3.4(ii) and (i), we have $\alpha\left(x^{n} \rightarrow x^{2 n}\right) \cap \alpha\left(x^{2 n} \rightarrow y\right) \subseteq \alpha\left(x^{n} \rightarrow y\right)$. By (iii), $\alpha\left(x^{n} \rightarrow\right.$ $\left.x^{2 n}\right)=\alpha(1)$, then $\alpha\left(x^{2 n} \rightarrow y\right) \subseteq \alpha\left(x^{n} \rightarrow y\right)$.

(iv) $\Rightarrow$ (i) Let $x, y, z \in H$ and $n \in \mathbb{N}$ such that $x^{n} \rightarrow(y \rightarrow$ $z), x^{n} \rightarrow y \in i_{H}(\alpha, \gamma)$. Then by Proposition 2.2(viii),

$$
\begin{aligned}
\left(x^{n} \rightarrow y\right) \odot\left(y \rightarrow\left(x^{n} \rightarrow z\right)\right) & \leq x^{n} \rightarrow\left(x^{n} \rightarrow z\right) \\
& =x^{2 n} \rightarrow z .
\end{aligned}
$$

Since $(\alpha, H)$ be an int-soft filter of $H$, by Corollary 3.4 (ii) and (i), we have $\alpha\left(x^{n} \rightarrow y\right) \cap \alpha\left(y \rightarrow\left(x^{n} \rightarrow z\right)\right) \subseteq \alpha\left(x^{2 n} \rightarrow z\right)$. Then by (iv), it is clear that

$$
\begin{aligned}
\alpha\left(x^{n} \rightarrow y\right) \cap \alpha\left(y \rightarrow\left(x^{n} \rightarrow z\right)\right) & \subseteq \alpha\left(x^{2 n} \rightarrow z\right) \\
& \subseteq \alpha\left(x^{n} \rightarrow z\right) .
\end{aligned}
$$

Hence, $x^{n} \rightarrow z \in i_{H}(\alpha, \gamma)$. Therefore, $(\alpha, H) \in I N I(\mathcal{H})$.

Corollary 4.6. If $(\alpha, H)$ is an int-soft filter of $H$ such that, for any $x \in H, x^{2}=x$, then $(\alpha, H) \in I N I(\mathcal{H})$.

Proof. Let $x \in H$. Since $x^{2}=x$, we have $x^{n}=x^{2 n}$, then $x^{n} \rightarrow x^{2 n}=1$, and so $\alpha\left(x^{n} \rightarrow x^{2 n}\right)=\alpha(1)$. Thus by Theorem 4.5. $(\alpha, H) \in \operatorname{INI}(\mathcal{H})$.

Theorem 4.7. Let $(\alpha, H)$ be an int-soft filter of $H$. Then $(\alpha, H) \in \operatorname{INI}(\mathcal{H})$ if and only if for any $x, y, z \in H$ and $n \in \mathbb{N}$, $\alpha\left(x^{n} \rightarrow(y \rightarrow z)\right) \subseteq \alpha\left(\left(x^{n} \rightarrow y\right) \rightarrow\left(x^{n} \rightarrow z\right)\right)$.

Proof. Suppose $(\alpha, H) \in \operatorname{INI}(\mathcal{H})$. Then by Proposition 2.2 (viii) and (vii), for any $x, y, z \in H$ and $n \in \mathbb{N}$, we have:

$$
\begin{aligned}
x^{n} \rightarrow(y \rightarrow z) & \leq x^{n} \rightarrow\left(\left(x^{n} \rightarrow y\right) \rightarrow\left(x^{n} \rightarrow z\right)\right) \\
& =x^{n} \rightarrow\left(x^{n} \rightarrow\left(\left(x^{n} \rightarrow y\right) \rightarrow z\right)\right) \\
& =x^{2 n} \rightarrow\left(\left(x^{n} \rightarrow y\right) \rightarrow z\right) .
\end{aligned}
$$

Also, by Proposition 2.2(viii),

$$
\begin{aligned}
& x^{2 n} \rightarrow\left(\left(x^{n} \rightarrow y\right) \rightarrow z\right) \\
& \leq\left(x^{n} \rightarrow x^{2 n}\right) \rightarrow\left(x^{n} \rightarrow\left(\left(x^{n} \rightarrow y\right) \rightarrow z\right)\right) .
\end{aligned}
$$

Since $(\alpha, H) \in \operatorname{INI}(\mathcal{H})$, by Theorem 4.3 , Corollary 3.4 i) and Theorem 4.5 (iii), we get that

$$
\begin{aligned}
& \alpha\left(x^{n} \rightarrow(y \rightarrow z)\right) \\
& \subseteq \alpha\left(x^{2 n} \rightarrow\left(\left(x^{n} \rightarrow y\right) \rightarrow z\right)\right. \\
& \subseteq \alpha\left(\left(x^{n} \rightarrow x^{2 n}\right) \rightarrow\left(\left(x^{n} \rightarrow\left(\left(x^{n} \rightarrow y\right) \rightarrow z\right)\right)\right)\right. \\
& \quad \cap \alpha\left(x^{n} \rightarrow x^{2 n}\right)
\end{aligned}
$$




$$
\subseteq \alpha\left(\left(x^{n} \rightarrow y\right) \rightarrow\left(x^{n} \rightarrow z\right)\right) .
$$

Conversely, let $x \in H$ and $n \in \mathbb{N}$. Since $x^{n} \rightarrow\left(x^{n} \rightarrow x^{2 n}\right)=$ 1 , by assumption, we have

$$
\begin{aligned}
\alpha(1) & =\alpha\left(x^{n} \rightarrow\left(x^{n} \rightarrow x^{2 n}\right)\right) \\
& \subseteq \alpha\left(\left(x^{n} \rightarrow x^{n}\right) \rightarrow\left(x^{n} \rightarrow x^{2 n}\right)\right) \\
& =\alpha\left(x^{n} \rightarrow x^{2 n}\right) .
\end{aligned}
$$

Then $\alpha\left(x^{n} \rightarrow x^{2 n}\right)=\alpha(1)$. Hence, by Theorem $4.5,(\alpha, H) \in$ $\operatorname{INI}(\mathcal{H})$.

Notation. Let $(\alpha, H)$ and $(\beta, H)$ be two int-soft filters of $H$ over $U$. If for any $x \in H, \alpha(x) \subseteq \beta(x)$, then we denoted it by $\alpha \leq \beta$.

Theorem 4.8. Let $(\alpha, H)$ and $(\beta, H)$ be two int-soft filters of $H$ over $U$ such that $\alpha \leq \beta$ and $\alpha(1)=\beta(1)$. If $(\alpha, H) \in$ $\operatorname{INI}(\mathcal{H})$, then $(\beta, H) \in \operatorname{INI}(\mathcal{H})$.

Proof. By Theorem 4.5, for any $x \in H$ and $n \in \mathbb{N}$, we have, $\beta(1)=\alpha(1) \subseteq \alpha\left(x^{n} \rightarrow x^{2 n}\right) \subseteq \beta\left(x^{n} \rightarrow x^{2 n}\right)$. Then $\beta\left(x^{n} \rightarrow x^{2 n}\right)=\beta(1)$. Hence, by Theorem 4.5. $(\beta, H) \in$ $\operatorname{INI}(\mathcal{H})$.

Theorem 4.9. If $(\alpha, H) \in \operatorname{INI}(\mathcal{H})$, then it is an int-soft $(n+1)$ fold implicative filter of $H$.

Proof. Let $(\alpha, H) \in \operatorname{INI}(\mathcal{H})$. Then by Theorem 4.7, for any $x, y \in H$, we have,

$$
\begin{aligned}
\alpha\left(x^{2(n+1)} \rightarrow y\right) & =\alpha\left(x^{n+1} \rightarrow\left(x^{n+1} \rightarrow y\right)\right) \\
& \subseteq \alpha\left(\left(x^{n+1} \rightarrow x^{n+1}\right) \rightarrow\left(x^{n+1} \rightarrow y\right)\right) \\
& \subseteq \alpha\left(x^{n+1} \rightarrow y\right) .
\end{aligned}
$$

Then by Theorem 4.5, $(\alpha, H)$ is an int-soft $(n+1)$-fold implicative filter of $H$.

Theorem 4.10. Let $(\alpha, H) \in \operatorname{INI}(\mathcal{H})$. Then every filter of $\underline{\underline{\underline{\underline{H}}}}$ is an implicative filter.

Proof. Let $(\alpha, H) \in \operatorname{INI}(\mathcal{H})$. Then by Theorem 4.3. $(\alpha, H)$ is an int-soft filter of $H$. Thus, by Theorem $3.7, \frac{H}{\equiv}$ is welldefine. Moreover, for any $x \in H$, it is clear that $x^{2 n} \rightarrow$ $x^{n}=1$, and so $\alpha\left(x^{2 n} \rightarrow x^{n}\right)=\alpha(1)$. Also, by Theorem 4.5 $\alpha\left(x^{n} \rightarrow x^{2 n}\right)=\alpha(1)$. Thus, $\left[x^{n}\right]=\left[x^{2 n}\right]$, and so $[x]=\left[x^{2}\right]$. By [?, Theorem 4.15], since class [1] is an int-soft filter of $H$ and for any $x \in H,\left[x^{2}\right]=[x]$, then [1] is an implicative filter of $H$. Hence, by [?, Theorem 4.10], since, for any filter $G$ of
$\frac{H}{\equiv(\alpha, H)}$ and $[1] \subseteq G$, we get that $\mathrm{G}$ is an int-soft implicative filter of $\frac{H}{\equiv_{(\alpha, H)}}$.

Theorem 4.11. Let $(\alpha, H)$ be an int-soft filter of $H$. Then $(\alpha, H)$ is an int-soft implicative filter of $H$ if and only if $\frac{H}{\equiv_{(\alpha, H)}}$ is a Brouwerian semilattice.

Proof. $(\Rightarrow)$ Let $(\alpha, H)$ be an int-soft filter of $H$. Then by Theorem 3.7. $\frac{H}{\equiv_{(\alpha, H)}}$ is well-defined. Since $\frac{H}{\equiv_{(\alpha, H)}}$ is a hoop, then it is clear that $\frac{H}{\equiv_{(\alpha, H)}}$ is an $\wedge$-semilattice. Now, it is enough to prove that for all $x, y, z \in H,[x]_{(\alpha, H)} \wedge[y]_{(\alpha, H)} \leq[z]_{(\alpha, H)}$ if and only if $[x]_{(\alpha, H)} \leq[y]_{(\alpha, H)} \rightsquigarrow[z]_{(\alpha, H)}$. Let $[x]_{(\alpha, H)} \wedge$ $[y]_{(\alpha, H)} \leq[z]_{(\alpha, H)}$. Then by Proposition 2.2 (iii),

$$
[x]_{(\alpha, H)} \otimes[y]_{(\alpha, H)} \leq[x]_{(\alpha, H)} \wedge[y]_{(\alpha, H)} \leq[z]_{(\alpha, H)} .
$$

Thus, $[x]_{(\alpha, H)} \otimes[y]_{(\alpha, H)} \leq[z]_{(\alpha, H)}$. Since $\frac{H}{\equiv_{(\alpha, H)}}$ is a hoop, then by Proposition 2.2 $(\mathrm{i}),[x]_{(\alpha, H)} \leq[y]_{(\alpha, H)} \rightsquigarrow[z]_{(\alpha, H)}$.

Conversely, suppose $[x]_{(\alpha, H)} \leq[y]_{(\alpha, H)} \rightsquigarrow[z]_{(\alpha, H)}$. Then by Theorem 3.7 $\alpha(x \rightarrow(y \rightarrow z))=\alpha(1)$, and so $\alpha(x \rightarrow$ $(y \rightarrow z)) \geq \alpha(1)$. Since $(\alpha, H)$ is an int-soft implicative filter of $H$, then by Theorem 4.7

$$
\begin{aligned}
\alpha(1) & \subseteq \alpha(x \rightarrow(y \rightarrow z)) \\
& \subseteq \alpha((x \rightarrow y) \rightarrow(x \rightarrow z)) .
\end{aligned}
$$

Thus, $[x \rightarrow y]_{(\alpha, H)} \leq[x \rightarrow z]_{(\alpha, H)}$ and so

$$
[x]_{(\alpha, H)} \rightsquigarrow[y]_{(\alpha, H)} \leq[x]_{(\alpha, H)} \rightsquigarrow[z]_{(\alpha, H)} .
$$

Since $\frac{H}{\equiv_{(\alpha, H)}}$ is a hoop, then

$$
\begin{aligned}
{[x]_{(\alpha, H)} \wedge[y]_{(\alpha, H)} } & \leq[x]_{(\alpha, H)} \otimes\left([x]_{(\alpha, H)} \rightsquigarrow[y]_{(\alpha, H)}\right) \\
& \leq[z]_{(\alpha, H)} .
\end{aligned}
$$

Therefore, $\frac{H}{\equiv_{(\alpha, H)}}$ is a Brouwerian semilattice.

$(\Leftarrow)$ Since $(\alpha, H)$ is an int-soft filter of $H$, then it is clear that $\alpha(x) \subseteq \alpha(1)$, for all $x \in H$. By assumption, $\frac{H}{\equiv_{(\alpha, H)}}$ is a Brouwerian semilattice, define $[x]_{(\alpha, H)} \otimes[y]_{(\alpha, H)}=[x]_{(\alpha, H)} \wedge$ $[y]_{(\alpha, H)}$, for all $x, y \in H$. Since $[x]_{(\alpha, H)} \leq[x]_{(\alpha, H)}$, then we have $[x]_{(\alpha, H)} \leq[x]_{(\alpha, H)} \wedge[x]_{(\alpha, H)}=[x]_{(\alpha, H)} \otimes[x]_{(\alpha, H)}=$ $\left[x^{2}\right]_{(\alpha, H)}$. So $[x]_{(\alpha, H)} \leq\left[x^{2}\right]_{(\alpha, H)}$. Then by Theorem 3.7 $\alpha\left(x \rightarrow x^{2}\right)=\alpha(1)$. Hence, by Theorem 4.3 (iii), $(\alpha, H)$ is an int-soft implicative filter of $H$. 


\section{Int-Soft n-Fold Positive Implicative Filters}

In this section, we introduce the notion of int-soft n-fold positive implicative filter and int-soft $n$-fold fantastic filter of hoop and investigate some properties and equivalence definitions of them. And we prove that every int-soft n-fold positive implicative filter is an int-soft fantastic filter.

Definition 5.1. A soft set $(\alpha, H)$ is an int-soft $n$-fold positive implicative filter of $H$ over $U$ if, for any $\gamma \in \mathcal{P}(U), \gamma$-inclusive set $\emptyset \neq i_{H}(\alpha, \gamma)$ of $(\alpha, H)$ is an $\mathrm{n}$-fold positive implicative filter of $H$. The set of all int-soft $\mathrm{n}$-fold positive implicative filters of $H$ is denoted by $\operatorname{INPI}(\mathcal{H})$.

Example 5.2. According to Example 3.2 it is clear that $(\alpha, H)$ is an int-soft n-fold positive implicative filter of $H$ over $U$.

Theorem 5.3. Every $(\alpha, H) \in \operatorname{INPI}(\mathcal{H})$ is an int-soft filter of $H$.

Proof. Let $(\alpha, H) \in \operatorname{INPI}(\mathcal{H})$. Since $i_{H}(\alpha, \gamma) \neq \emptyset$, we have $x \in i_{H}(\alpha, \gamma)$ and we suppose $\gamma=\alpha(x)$. Since $(\alpha, H) \in$ $\operatorname{INPI}(\mathcal{H})$, we have $i_{H}(\alpha, \gamma)$ is an $\mathrm{n}$-fold positive implicative filter of $H$, then $1 \in i_{H}(\alpha, \gamma)$ and so $\alpha(x)=\gamma \subseteq \alpha(1)$. Now, let $x, y, z \in H$ such that $x, x \rightarrow y \in i_{H}(\alpha, \gamma)$. Suppose $\gamma=\alpha(x) \cap \alpha(x \rightarrow y)$. Since $i_{H}(\alpha, \gamma)$ is an $\mathrm{n}$-fold positive implicative filter of $H$, for any $n \in \mathbb{N}$, we have $x, x \rightarrow\left(\left(y^{n} \rightarrow\right.\right.$ 1) $\rightarrow y) \in i_{H}(\alpha, \gamma)$, then $y \in i_{H}(\alpha, \gamma)$, and so

$$
\begin{aligned}
\gamma & =\alpha(x) \cap \alpha(x \rightarrow y)=\alpha(x) \cap \alpha\left(x \rightarrow\left(\left(y^{n} \rightarrow 1\right) \rightarrow y\right)\right) \\
& \subseteq \alpha(y) .
\end{aligned}
$$

Hence, $(\alpha, H)$ is an int-soft filter of $H$.

Example 5.4. According to Example 3.2, $H$ is a bounded hoop. Let define $\alpha(1)=\mathbb{Z}, \alpha(a)=2 \mathbb{Z}, \alpha(b)=4 \mathbb{Z}, \alpha(c)=2 \mathbb{Z}$ and $\alpha(0)=4 \mathbb{Z}$. Then by routine calculations, we can see that $(\alpha, H)$ is an int-soft $\mathbf{n}$-fold positive implicative filter but it is not an int-soft filter of $H$ over $U$. Because

$$
\begin{aligned}
\mathbb{Z} & =\alpha(1)=\alpha\left(1 \rightarrow\left(\left(c^{n} \rightarrow 0\right) \rightarrow c\right)\right) \cap \alpha(1) \nsubseteq \propto \alpha(c) \\
& =2 \mathbb{Z} .
\end{aligned}
$$

Theorem 5.5. Let $(\alpha, H)$ be an int-soft filter of $H$ over $U$. Then, for any $x, y, z \in H$ and $n \in \mathbb{N}$, the following conditions are equivalent:

(i) $(\alpha, H) \in \operatorname{INPI}(\mathcal{H})$,

(ii) $\alpha(x) \subseteq \alpha(1)$ and $\alpha\left(x \rightarrow\left(\left(y^{n} \rightarrow z\right) \rightarrow y\right)\right) \cap \alpha(x) \subseteq$ $\alpha(y)$, (iii) $\alpha\left(\left(x^{n} \rightarrow y\right) \rightarrow x\right) \subseteq \alpha(x)$,

(iv) $\alpha\left(\left(x^{n} \rightarrow 0\right) \rightarrow x\right) \subseteq \alpha(x)$.

Proof. (i) $\Rightarrow$ (ii) Let $(\alpha, H) \in \operatorname{INPI}(\mathcal{H}), x, y, z \in H$ and $n \in$ $\mathbb{N}$. Define $\gamma=\alpha(x) \cap \alpha\left(x \rightarrow\left(\left(y^{n} \rightarrow z\right) \rightarrow y\right)\right)$. Then $x \rightarrow$ $\left(\left(y^{n} \rightarrow z\right) \rightarrow y\right), x \in i_{H}(\alpha, \gamma)$. Moreover, since $i_{H}(\alpha, \gamma)$ is an n-fold positive implicative filter of $H, y \in i_{H}(\alpha, \gamma)$. Then, $\gamma=\alpha(x) \cap \alpha\left(x \rightarrow\left(\left(y^{n} \rightarrow z\right) \rightarrow y\right)\right) \subseteq \alpha(y)$.

(ii) $\Rightarrow$ (iii) By (ii), we have $\alpha(1) \cap \alpha\left(1 \rightarrow\left(\left(x^{n} \rightarrow y\right) \rightarrow\right.\right.$ $x)) \subseteq \alpha(x)$.

(iii) $\Rightarrow$ (iv) It is enough let $y=0$ in (iii).

(iv) $\Rightarrow$ (i) Since $(\alpha, H)$ is an int-soft filter of $H$, for any $x, y, z \in H$ and $n \in \mathbb{N}$,

$$
\left.\alpha\left(x \rightarrow\left(\left(y^{n} \rightarrow z\right) \rightarrow y\right)\right) \cap \alpha(x) \subseteq \alpha\left(\left(y^{n} \rightarrow z\right) \rightarrow y\right)\right) .
$$

Moreover, since $0 \leq z$, by Proposition 2.2. (vii), $y^{n} \rightarrow 0 \leq$ $y^{n} \rightarrow z$ and so $\left(y^{n} \rightarrow z\right) \rightarrow y \leq\left(y^{n} \rightarrow 0\right) \rightarrow y$. Then $\left(\left(y^{n} \rightarrow z\right) \rightarrow y\right) \rightarrow\left(\left(y^{n} \rightarrow 0\right) \rightarrow y\right)=1$ and by Corollary 3.4. $\left.\alpha\left(\left(y^{n} \rightarrow z\right) \rightarrow y\right)\right) \subseteq \alpha\left(\left(y^{n} \rightarrow 0\right) \rightarrow y\right) \subseteq \alpha(y)$. Hence, by (iv), $(\alpha, H) \in \operatorname{INPI}(\mathcal{H})$.

Theorem 5.6. Let $(\alpha, H) \in \operatorname{INPI}(\mathcal{H})$. Then $(\alpha, H) \in \operatorname{INI}(\mathcal{H})$.

Proof. Let $(\alpha, H) \in \operatorname{INPI}(\mathcal{H})$. Then by Definition 5.1, $i_{H}(\alpha, \gamma)$ is an $\mathrm{n}$-fold positive implicative filter of $H$, thus, by [?, Theorem 4.8], every n-fold positive implicative filter of $H$ is an n-fold implicative filter of $H$, so $i_{H}(\alpha, \gamma)$ is an n-fold implicative filter of $H$. Hence, $(\alpha, H)$ is an int-soft n-fold implicative filter of $H$.

Theorem 5.7. Let $(\alpha, H) \in \operatorname{INPI}(\mathcal{H})$. Then $(\alpha, H)$ is an intsoft $(\mathrm{n}+1)$-fold positive implicative filter of $H$ over $U$.

Proof. Let $x \in H$ and $n \in \mathbb{N}$. Since $x^{n+1} \leq x^{n}$, by Proposition 2.2.vii), $x^{n} \rightarrow 0 \leq x^{n+1} \rightarrow 0$, and so $\left(x^{n+1} \rightarrow 0\right) \rightarrow$ $x \leq\left(x^{n} \rightarrow 0\right) \rightarrow x$. Since $(\alpha, H) \in \operatorname{INPI}(\mathcal{H})$, by Theorem 5.3 and Corollary 3.4 , we have

$$
\alpha\left(\left(x^{n+1} \rightarrow 0\right) \rightarrow x\right) \subseteq \alpha\left(\left(x^{n} \rightarrow 0\right) \rightarrow x\right) \subseteq \alpha(x) .
$$

Hence, by Theorem 5.5. $(\alpha, H)$ is an int-soft $(\mathrm{n}+1)$-fold positive implicative filter of $H$.

Definition 5.8. A soft set $(\alpha, H)$ is called an int-soft $n$-fold fantastic filter of $H$ over $U$ if, for all $\gamma \in \mathcal{P}(U), \gamma$-inclusive set $\emptyset \neq i_{H}(\alpha, \gamma)$ of $(\alpha, H)$ is a $\mathrm{n}$-fold fantastic filter of $H$.

The set of all int-soft $\mathrm{n}$-fold fantastic filters of $H$ is denoted by $\operatorname{INFF}(\mathcal{H})$. 
Example 5.9. According to Example 3.2 it is clear that $(\alpha, H)$ is an int-soft $\mathrm{n}$-fold fantastic filter of $H$ over $U$.

Theorem 5.10. Let $(\alpha, H) \in \operatorname{INFF}(\mathcal{H})$. Then $(\alpha, H)$ is an int-soft n-fold filter of $H$.

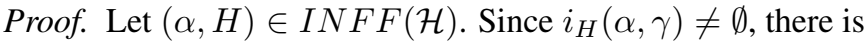
$x \in i_{H}(\alpha, \gamma)$. Define $\gamma=\alpha(x)$. Since $i_{H}(\alpha, \gamma)$ is an $\mathrm{n}$-fold fantastic filter of $H, 1 \in i_{H}(\alpha, \gamma)$ and so $\alpha(x)=\gamma \subseteq \alpha(1)$. Now, let $x, y \in H$ such that $x, x \rightarrow y \in i_{H}(\alpha, \gamma)$. Then $\gamma=\alpha(x) \cap \alpha(x \rightarrow y)$. Since $i_{H}(\alpha, \gamma)$ is an $\mathrm{n}$-fold fantastic filter of $H,\left(\left(y^{n} \rightarrow 1\right) \rightarrow 1\right) \rightarrow y \in i_{H}(\alpha, \gamma)$. Thus,

$$
\begin{aligned}
\alpha(x) \cap \alpha(x \rightarrow y) & =\alpha(x) \cap \alpha(x \rightarrow(1 \rightarrow y)) \\
& \subseteq \alpha\left(\left(\left(y^{n} \rightarrow 1\right) \rightarrow 1\right) \rightarrow y\right) \\
& =\alpha(y) .
\end{aligned}
$$

Hence, $i_{H}(\alpha, \gamma)$ is an int-soft n-fold filter of $H$.

Example 5.11. According to Example 5.4. $(\alpha, H)$ is an int-soft filter of $H$ over $U$ but it is not an int-soft $\mathrm{n}$-fold fantastic filter of $H$. Because,

$$
\begin{aligned}
\mathbb{Z} & =\alpha(1) \\
& =\alpha(1) \cap \alpha(1 \rightarrow(0 \rightarrow c)) \\
& \nsubseteq \alpha\left(\left(\left(c^{n} \rightarrow 0\right) \rightarrow 0\right) \rightarrow c\right) \\
& =2 \mathbb{Z} .
\end{aligned}
$$

Theorem 5.12. Let $(\alpha, H)$ be a soft set of $H$. Then, for any $x, y, z \in H$, the following statements are equivalent:

(i) $(\alpha, H) \in \operatorname{INFF}(\mathcal{H})$,

(ii) $\alpha(z) \cap \alpha(z \rightarrow(y \rightarrow x)) \subseteq \alpha\left(\left(\left(x^{n} \rightarrow y\right) \rightarrow y\right) \rightarrow x\right)$, $\alpha(x) \subseteq \alpha(1)$,

(iii) $\alpha(y \rightarrow x) \subseteq \alpha\left(\left(\left(x^{n} \rightarrow y\right) \rightarrow y\right) \rightarrow x\right), \alpha(x) \subseteq \alpha(1)$.

Proof. (i) $\Rightarrow$ (ii) Suppose $(\alpha, H) \in \operatorname{INFF}(\mathcal{H}), x, y, z \in H$ and $\gamma \in \mathcal{P}(U)$ be a $\gamma$-inclusive set. Let $\gamma_{f}=\alpha(z) \cap \alpha(z \rightarrow$ $(y \rightarrow x))$. Then $\gamma_{f} \subseteq \alpha(z)$ and $\gamma_{f} \subseteq \alpha(z \rightarrow(y \rightarrow x))$, and so $z \in i_{H}(\alpha, \gamma)$ and $z \rightarrow(y \rightarrow x) \in i_{H}(\alpha, \gamma)$. Since $i_{H}(\alpha, \gamma)$ is an $\mathrm{n}$-fold fantastic filter of $H$, we have $\left(\left(x^{n} \rightarrow y\right) \rightarrow y\right) \rightarrow$ $x \in i_{H}(\alpha, \gamma)$, thus $\gamma_{f} \subseteq \alpha\left(\left(\left(x^{n} \rightarrow y\right) \rightarrow y\right) \rightarrow x\right)$. Hence,

$$
\alpha(z) \cap \alpha(z \rightarrow(y \rightarrow x)) \subseteq \alpha\left(\left(\left(x^{n} \rightarrow y\right) \rightarrow y\right) \rightarrow x\right) .
$$

Let $\alpha(x)=\gamma$. Then $x \in i_{H}(\alpha, \gamma)$. Since $i_{H}(\alpha, \gamma)$ is an int-soft $\mathrm{n}$-fold fantastic filter, we have $1 \in i_{H}(\alpha, \gamma)$ and so $\gamma \subseteq \alpha(1)$. Hence, $\alpha(x) \subseteq \alpha(1)$.

(ii) $\Rightarrow$ (iii) It is enough to let $z=1$. (iii) $\Rightarrow$ (i) Let $x \in i_{H}(\alpha, \gamma)$. Then $\gamma \subseteq \alpha(x)$, by assumption $\alpha(x) \subseteq \alpha(1)$. Thus $\gamma \subseteq \alpha(1)$, and so $1 \in i_{H}(\alpha, \gamma)$. Now, suppose $x, y \in H$ such that $y \rightarrow x \in i_{H}(\alpha, \gamma)$, then $\gamma \subseteq$ $\alpha(y \rightarrow x)$. Since $\alpha(y \rightarrow x) \subseteq \alpha\left(\left(\left(x^{n} \rightarrow y\right) \rightarrow y\right) \rightarrow x\right)$, we get that $\gamma \subseteq \alpha\left(\left(\left(x^{n} \rightarrow y\right) \rightarrow y\right) \rightarrow x\right)$. Hence, $\left(\left(x^{n} \rightarrow y\right) \rightarrow\right.$ $y) \rightarrow x \in i_{H}(\alpha, \gamma)$. Hence, $i_{H}(\alpha, \gamma)$ is an $\mathbf{n}$-fold fantastic filter of $H$. Therefore, $(\alpha, H) \in I N F F(\mathcal{H})$.

Theorem 5.13. Every int-soft $\mathrm{n}$-fold fantastic filter of $H$ is an int-soft $(\mathrm{n}+1)$-fold fantastic filter of $H$.

Proof. Let $(\alpha, H) \in \operatorname{INFF}(\mathcal{H}), x \in H$ and $n \in \mathbb{N}$. By Proposition 2.2(iii) and (vii), $x^{n+1} \leq x^{n}$, then $x^{n} \rightarrow y \leq$ $x^{n+1} \rightarrow y$. By repeating this method, we have $\left(x^{n+1} \rightarrow y\right) \rightarrow$ $y \leq\left(x^{n} \rightarrow y\right) \rightarrow y$ and so

$$
\left(\left(x^{n} \rightarrow y\right) \rightarrow y\right) \rightarrow x \leq\left(\left(x^{n+1} \rightarrow y\right) \rightarrow y\right) \rightarrow x .
$$

Since $(\alpha, H)$ is an int-soft $\mathbf{n}$-fold fantastic filter of $H$, by Theorem 5.10, $(\alpha, H)$ is an int-soft $\mathrm{n}$-fold filter of $H$ and by Theorem 5.12

$$
\begin{aligned}
\alpha(y \rightarrow x) & \subseteq \alpha\left(\left(\left(x^{n} \rightarrow y\right) \rightarrow y\right) \rightarrow x\right) \\
& \subseteq \alpha\left(\left(\left(x^{n+1} \rightarrow y\right) \rightarrow y\right) \rightarrow x\right) .
\end{aligned}
$$

Hence, by Theorem 5.12, $(\alpha, H)$ is an int-soft $(\mathrm{n}+1)$-fold fantastic filter of $H$.

Theorem 5.14. Let $(\alpha, H) \in I N P I(\mathcal{H})$. Then $(\alpha, H) \in$ $\operatorname{INFF}(\mathcal{H})$.

Proof. Let $x, y \in H$ and $n \in \mathbb{N}$. Then by Proposition 2.2(viii), we have $\left(\left(x^{n} \rightarrow y\right) \rightarrow y\right) \odot(y \rightarrow x) \leq\left(x^{n} \rightarrow y\right) \rightarrow x$ and so $y \rightarrow x \leq\left[\left(x^{n} \rightarrow y\right) \rightarrow y\right] \rightarrow\left[\left(x^{n} \rightarrow y\right) \rightarrow x\right]$ Also, by Proposition 2.2(v), $x^{n} \leq\left(\left(x^{n} \rightarrow y\right) \rightarrow y\right) \rightarrow x$. Then by Proposition 2.2 (viii), $\left.\left(\left(x^{n} \rightarrow y\right) \rightarrow y\right) \rightarrow x\right) \rightarrow y \leq x^{n} \rightarrow y$ and so $\left.x^{n} \rightarrow y\right) \rightarrow\left(\left(\left(x^{n} \rightarrow y\right) \rightarrow y\right) \rightarrow x\right) \leq\left(\left(\left(x^{n} \rightarrow\right.\right.\right.$ $y) \rightarrow y) \rightarrow x) \rightarrow y) \rightarrow\left(\left(\left(x^{n} \rightarrow y\right) \rightarrow y\right) \rightarrow x\right)$. Hence, $\left.y \rightarrow x \leq\left(\left(\left(x^{n} \rightarrow y\right) \rightarrow y\right) \rightarrow x\right) \rightarrow y\right) \rightarrow\left(\left(\left(x^{n} \rightarrow y\right) \rightarrow\right.\right.$ $y) \rightarrow x)$. Since $(\alpha, H) \in I N P I(\mathcal{H})$, by Theorem 5.3. $(\alpha, H)$ is an int-soft filter of $H$. Then by Corollary $3.4(\mathrm{i}), \alpha(y \rightarrow x) \subseteq$ $\left.\alpha\left(\left(\left(\left(x^{n} \rightarrow y\right) \rightarrow y\right) \rightarrow x\right) \rightarrow y\right) \rightarrow\left(\left(\left(x^{n} \rightarrow y\right) \rightarrow y\right) \rightarrow x\right)\right)$ Moreover, since $(\alpha, H) \in I N P I(\mathcal{H})$, by Theorem 5.5(iii), we get that

$$
\begin{aligned}
& \alpha(y \rightarrow x) \\
& \left.\subseteq \alpha\left(\left(\left(\left(x^{n} \rightarrow y\right) \rightarrow y\right) \rightarrow x\right) \rightarrow y\right) \rightarrow\left(\left(\left(x^{n} \rightarrow y\right) \rightarrow y\right) \rightarrow x\right)\right) \\
& \subseteq \alpha\left(\left(\left(x^{n} \rightarrow y\right) \rightarrow y\right) \rightarrow x\right) .
\end{aligned}
$$


Hence, $\alpha(y \rightarrow x) \subseteq \alpha\left(\left(\left(x^{n} \rightarrow y\right) \rightarrow y\right) \rightarrow x\right)$. Therefore, by Theorem 5.12, $(\alpha, H) \in \operatorname{INFF}(\mathcal{H})$.

\section{Conclusions}

In this paper, the notion of int-soft filter in hoops were introduced and related properties investigated. Characterizations of int-soft filters discussed and a congruence relation was introduced by using int-soft filter and was proved the quotient is a hoop. Also, the notions of int-soft $n$-fold (positive) implicative and int-soft n-fold fantastic filters of hoops were introduced, some equivalence definitions of them and relation between them studied. Moreover, it was proved that every int-soft n-fold positive implicative filter is an int-soft fantastic filter and int-soft implicative filter. Also, the quotient structure that is made by them was investigated and proved that $(\alpha, H)$ is an int-soft implicative filter of $H$ if and only if $\frac{H}{\equiv_{(\alpha, H)}}$ is a Brouwerian semilattice.

In the future works, int-soft of other filters such as prime, maximal, nodal and etc of hoops and the quotient structures can be study.

\section{References}

[1] B. Bosbach, "Komplementäre halbgruppen. axiomatik und arithmetic," Fundamenta Mathematicae, vol. 64, no. 3, pp. 257-287, 1969.

[2] B. Bosbach, "Komplementäre halbgruppen kongruenzen und quotienten," Fundamenta Mathematicae, vol. 69, no. 1, pp. 1-14, 1970.

[3] P. Agliano, I. M. Ferreirim, and F. Montagna, "Basic hoops: an algebraic study of continuous t-norms," Studia Logica, vol. 87, no. 1, pp. 73-98, 2007.https://doi.org/10. 1007/s11225-007-9078-1

[4] P. Hajek, Metamathematics of Fuzzy Logic. Berlin: Springer, 1998.

[5] A. Namdar, R. A. Borzooei, A. Borumand Saeid, and M. Aaly Kologani, "Some results in hoop algebras," Journal of Intelligent \& Fuzzy Systems, vol. 32, no. 3, pp. 18051813, 2017. https://doi.org/10.3233/JIFS-152553

[6] R. A. Borzooei and A. Kologani, "Filter theory of hoopalgebras," Journal of Advanced Research in Pure Mathematics, vol. 6, pp. 72-86, 2014.
[7] D. Molodtsov, "Soft set theory: first results," Computers \& Mathematics with Applications, vol. 37, no. 4-5, pp. 19-31, 1999. https://doi.org/10.1016/S0898-1221(99)00056-5

[8] P. K. Maji, A. R. Roy, and R. Biswas, "An application of soft sets in a decision making problem," Computers \& Mathematics with Applications, vol. 44, no. 8-9, pp. 1077-1083, 2002. https://doi.org/10.1016/S0898-1221(02) 00216-X

[9] P. K. Maji, R. Biswas, and A. Roy, "Soft set theory," Computers \& Mathematics with Applications, vol. 45, no. 4-5, pp. 555-562, 2003. https://doi.org/10.1016/S08981221(03)00016-6

[10] D. Chen, E. C. C. Tsang, D. S. Yeung, and X. Wang, "The parameterization reduction of soft sets and its applications," Computers \& Mathematics with Applications, vol. 49, no. 5-6, pp. 757-763, 2005. https://doi.org/10.1016/j. camwa.2004.10.036

[11] F. Feng, "Soft rough sets applied to multicriteria group decision making," Annals of Fuzzy Mathematics and Informatics, vol. 2, no. 1, pp. 69-80, 2011.

[12] H. Aktas and N. Cagman, "Soft sets and soft groups," Information Sciences, vol. 177, no. 13, pp. 2726-2735, 2007. https://doi.org/10.1016/j.ins.2006.12.008

[13] R. A. Borzooei, M. Mobini, and M. M. Ebrahimi, "The category of soft sets," Journal of Intelligent \& Fuzzy Systems, vol. 28, no. 1, pp. 157-167, 2015. https://doi.org/10. 3233/IFS-141286

[14] Y. B. Jun, "Soft Bck/Bci-algebras," Computers \& Mathematics with Applications, vol. 56, no. 5, pp. 1408-1413, 2008. https://doi.org/10.1016/j.camwa.2008.02.035

[15] Y. B. Jun, K. J. Lee, and C. H. Park, "Fuzzy soft set theory applied to BCK/BCI-algebras," Computers \& Mathematics with Applications, vol. 59, no. 9, pp. 3180-3192, 2010 https://doi.org/10.1016/j.camwa.2010.03.004

[16] P. K. Maji, P. Biswas, and A. R. Roy, "A fuzzy soft sets," Journal of Fuzzy Mathematics, vol. 9, no. 3, pp. 589-602 2001.

[17] C. Luo, X. Xin, and P. He, "n-fold (positive) implicative filters of hoops," Italian Journal of Pure and Applied Mathematics, vol. 38, pp. 631-642, 2017. 


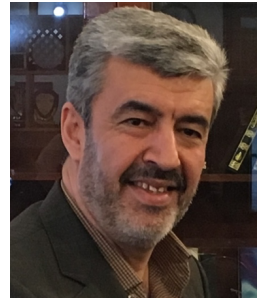

R. A. Borzooei is a full professor at the Shahid Beheshti University. He is currently an Managing Editor and fonder of the Iranian Journal of Fuzzy Systems, and was an editorial board 5 journals. He published more than 220 publications in journals on logical algebras, algebraic hyperstructuers, and fuzzy graph theory.

E-mail: borzooei@sub.ac.ir

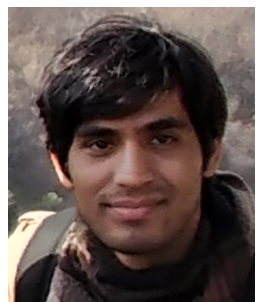

M. Sabetkish is a student at department of mathematics at Shahid Beheshti University, Iran. He has been working on research related to the soft and fuzzy logical algebras. He published more than 2 publications in journals on soft and fuzzy

logical algebras.

E-mail: m.sabetkish@sub.ac.ir

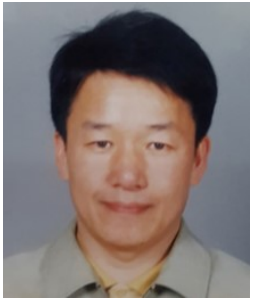

E. H. Roh is a full professor at the Department of Mathematics Education, Chinju National University of Education. He is currently an Editor of 4 journals. He published more than 230 publications in peerreviewed journals or conferences.

E-mail: ehroh9988@gmail.com

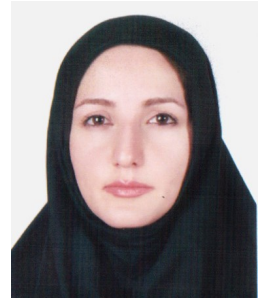

M. Aaly Kologani is a assistant professor at Hatef Higher Education Institute. He published more than 20 publications in peer-reviewed journals on logical algebras, algebraic hyperstructuers.

E-mail: mona4011@gmail.com 